$\overline{A E E T}$

ASOCIACIÓN ESPAÑOLA DE ECOLOGÍA TERRESTRE

\title{
Conservación y gestión cinegética del corzo en Andalucía
}

\author{
C. San José ${ }^{1, *}$, P. Fernández-Salguero ${ }^{2}$, I. Redondo ${ }^{3}$
}

(1) Red Andaluza de Estaciones de Referencia. Agencia de Medio Ambiente y Agua de Andalucía-Consejería de Agricultura, Pesca y Medio Ambiente, Junta de Andalucía. C/ Johan G. Gutenberg n¹, 41092 Sevilla, España.

(2) Departamento de Caza y Pesca. Servicio de Gestión del Medio Natural. Delegación Territorial en Córdoba. Consejería de Agricultura, Pesca y Medio Ambiente, Junta de Andalucía. C/ Tomás de Aquino, s/n. $7^{\text {a }}$ planta. Edificio Servicios Múltiples, 14071 Córdoba, España.

(3) Instituto Andaluz de Caza y Pesca Continental. Dirección General de Gestión del Medio Natural Consejería de Agricultura, Pesca y Medio Ambiente, Junta de Andalucía. Avda. Manuel Siurot 50, 41071 Sevilla, España.

* Autor de correspondencia: C. San José [csanjose@agenciamedioambienteyagua.es]

> Recibido el 28 de enero de 2012, aceptado el 26 de mayo de 2013

San José, C., Fernández-Salguero, P., Redondo, I. (2013). Conservación y gestión cinegética del corzo en Andalucía. Ecosistemas 22(2):2026. Doi.: 10.7818/ECOS.2013.22-2.04

Actualmente las poblaciones andaluzas de corzo se limitan a dos núcleos separados por el río Guadalquivir, el primero de ellos y más amenazado en Sierra Morena, entre las provincias de Ciudad Real, Córdoba y Jaén. El segundo, relativamente bien conservado, en las Sierras de Cádiz-Málaga. La desaparición del corzo de muchas áreas andaluzas ha sido debida fundamentalmente a la desaparición de los hábitats y los requerimientos básicos para la vida de esta especie, y al crecimiento de otras poblaciones de ungulados silvestres y domésticos. Los estudios llevados a cabo a lo largo de los últimos 25 años sobre el corzo andaluz de las Sierras de Cádiz-Málaga condujeron a la descripción de un ecotipo propio de estas sierras (con diferencias significativas a nivel morfológico y genético respecto a otras poblaciones españolas y europeas de corzo) y recientemente a su catalogación por el CIC (Consejo Internacional de la Caza) como un trofeo de caza singular. Partiendo de estas particularidades, la gestión del corzo en Andalucía se articula alrededor de dos objetivos: 1) conservar el ecotipo de corzo andaluz propio de las Sierras de Cádiz-Málaga, y 2) garantizar el buen estado de conservación del hábitat que ocupa la especie actualmente, así como mejorar áreas potencialmente buenas para su colonización natural o mediante reintroducciones.

Palabras clave: biodiversidad, corzo andaluz, ecosistemas mediterráneos, especies cinegéticas

San José, C., Fernández-Salguero, P., Redondo, I. (2013). Conservation and game management of roe deer in Andalusia. Ecosistemas 22(2):20-26. Doi.: 10.7818/ECOS.2013.22-2.04

At present roe deer distribution in Andalusia is limited to two population nuclei separated by the Guadalquivir River. The first one, more threatened by demographic extinction, is located in Sierra Morena, between Ciudad Real, Córdoba and Jaén provinces. The second one, relatively well conserved, is located in the mountainous zones of Cádiz-Málaga. The roe deer disappeared from many Andalusian areas mainly due to the disappearing of the suitable habitat for the species and to the increase of other wild and domestic ungulate populations. The studies carried out over the last 25 years on the Andalusian roe deer populations of Cádiz-Málaga revealed the existence of an ecotype native to the mountainous zones of these two Andalusian provinces, which presents significant differences on a morphological and genetic level with respect to other Spanish and European roe deer populations, and which was recently catalogued by the CIC (International Council for Game and Wildlife Conservation) as a singular trophy. Taking into account all these particularities, roe deer management in Andalusia is focused on two main objectives: 1) the conservation of the Andalusian ecotype native to the mountainous areas of Cádiz-Málaga, and 2) guaranteeing a good level of conservation of the current Andalusian habitat suitable for roe deer, as well as improving the potentially optimal areas for natural colonization by roe deer or potential reintroductions of the species in Andalusia.

Key words: biodiversity, andalusian roe deer, mediterranean ecosystems, game species

\section{Introducción}

El corzo europeo (Capreolus capreolus L. 1758) es una especie propia de la región paleártica occidental, cuya área de distribución natural se extiende por la práctica totalidad del continente europeo. En la cuenca mediterránea este área se caracteriza por la fragmentación de las poblaciones y la disminución de la densidad en las zonas más meridionales.

Mientras la especie presenta preferentemente problemas de sobreabundancia en la mitad norte de España y gran parte de Europa, el corzo aparece catalogado como "Vulnerable" en el Libro Rojo de los Vertebrados de Andalucía (Braza et al. 2001), debido a la reducción y fragmentación de sus poblaciones en esta región, y a la exclusividad del ecotipo descrito para las sierras de Cádiz-Málaga, cuyas poblaciones constituyen el límite suroccidental de distribución del corzo a nivel mundial (Braza et al. 2004; Carranza 2010).

La relativamente baja abundancia del corzo en Andalucía hace que la gestión de la especie tenga un carácter fundamentalmente conservacionista, para garantizar la estabilidad de las poblaciones y potenciar su desarrollo y expansión colonizadora hacia nuevas áreas. Por otro lado, los estudios promovidos sobre la especie por la Junta de Andalucía desde finales de los años 80, y llevados a cabo por diferentes centros de investigación, han puesto de manifiesto la existencia de diferencias significativas entre los corzos más meridionales de las Sierras de Cádiz-Málaga y el resto de poblaciones de corzo de España y Europa, y han permitido conocer las 
adaptaciones de la especie a los hábitats mediterráneos propios de estas áreas, además de constituir la base sobre la que se ha apoyado la estrategia de conservación y gestión de la especie en Andalucía (Aragón 1993; Aragón et al. 1995a y b, 1998; Lorenzini et al. 2003; Cassinello et al. 2009).

Todo ello ha permitido afianzar el estatus poblacional del corzo en las provincias de Cádiz y Málaga, y recientemente iniciar la recuperación de territorios donde la especie ha mantenido una presencia mínima, como la Sierra de Andújar (Jaén), e incluso de áreas donde se extinguió en tiempos recientes (Menor y Prenda 2005), como la Sierra de Huétor en Granada o las Sierras de Tejeda, Almijara y Alhama, en el límite entre las provincias de Málaga y Granada.

\section{Caracterización, distribución y estatus de las poblaciones andaluzas de corzo}

Aunque hasta finales del siglo XIX la especie se hallaba presente en la mayoría de las sierras andaluzas (Braza et al. 2004; Menor y Prenda 2005), actualmente las poblaciones naturales de corzo se distribuyen en Andalucía en dos núcleos, separados por el río Guadalquivir: el primero de ellos y el más amenazado se localiza en Sierra Morena, a caballo entre las provincias de Ciudad Real, Córdoba y Jaén, y el segundo, relativamente abundante y bien conservado, habita en las Sierras de Cádiz-Málaga (San José 2007; Figs. 1 y 2). Aparte de estos dos núcleos poblacionales principales, el corzo está presente además en algunos cotos privados de caza de Córdoba, Sevilla y Huelva, donde en los últimos 10 años se han efectuado repoblaciones con la especie, y recientemente, en el marco del "Plan de Gestión y Reintroducción del Corzo en Andalucía" (ver punto "Objetivos y estrategia actual para la conservación y gestión del corzo en Andalucía"), se ha iniciado la implantación de tres nuevos núcleos de corzo en la Sierra de Andújar (Jaén, refuerzo de la población original de Sierra Morena), Sierra de Huétor (Granada, reintroducción) y Sierras de Tejeda-Almijara (Málaga, reintroducción) (Fig. 1).

La desaparición del corzo de muchas áreas andaluzas ha sido debida fundamentalmente a dos factores (Braza et al. 1994; Aragón et al. 1995b; Consejería de Medio Ambiente 2007):

- La desaparición de los hábitats y los requerimientos básicos para la vida de esta especie. El corzo es una especie de bosque, ramoneadora y que necesita una alta variedad en los componentes de su dieta, además de un nivel elevado de cobertura arbórea y arbustiva mezclada con claros y zonas abiertas de pastizal. Pero incluso más importantes que estos requerimientos de vegetación, son los requerimientos de agua, en concreto de fuentes y cursos de agua limpia, especialmente durante el verano.

- El crecimiento de las poblaciones de ciervo, la introducción de ungulados silvestres (gamo y muflón) y domésticos (fundamentalmente cabra), que compiten con ventaja frente al corzo, y el incremento de las poblaciones de cochino asilvestrado, que pueden predar sobre las crías de corzo y compiten por el espacio. Al ser territorial y de menor envergadura que otros ungulados silvestres y domésticos, el corzo se ve en desventaja a la hora de competir por espacio y alimento.

Los estudios llevados a cabo a lo largo de los últimos 25 años sobre el corzo andaluz de las Sierras de Cádiz-Málaga condujeron a la descripción científica de un ecotipo propio de estas sierras (Braza et al. 1994; Aragón et al. 1998; Lorenzini et al., 2003), con diferencias significativas a nivel morfológico y genético respecto a otras poblaciones españolas y europeas de corzo, y recientemente a su catalogación por el CIC (Consejo Internacional de la Caza) como un trofeo de caza singular (Resolución Consejo CIC - Bruselas - 17 noviembre 2009, refrendada en Asamblea Anual CIC - Dubrovnik - 6-9 mayo 2010).

Este ecotipo de corzo, conocido popularmente como "corzo morisco" (Fig. 3), se caracteriza por una coloración invariablemente gris a lo largo de todo el año, pequeño tamaño, dimorfismo sexual más acentuado (Aragón 1993; Aragón et al. 1995a), y cráneos más cortos y anchos con mandíbulas significativamente más cortas y

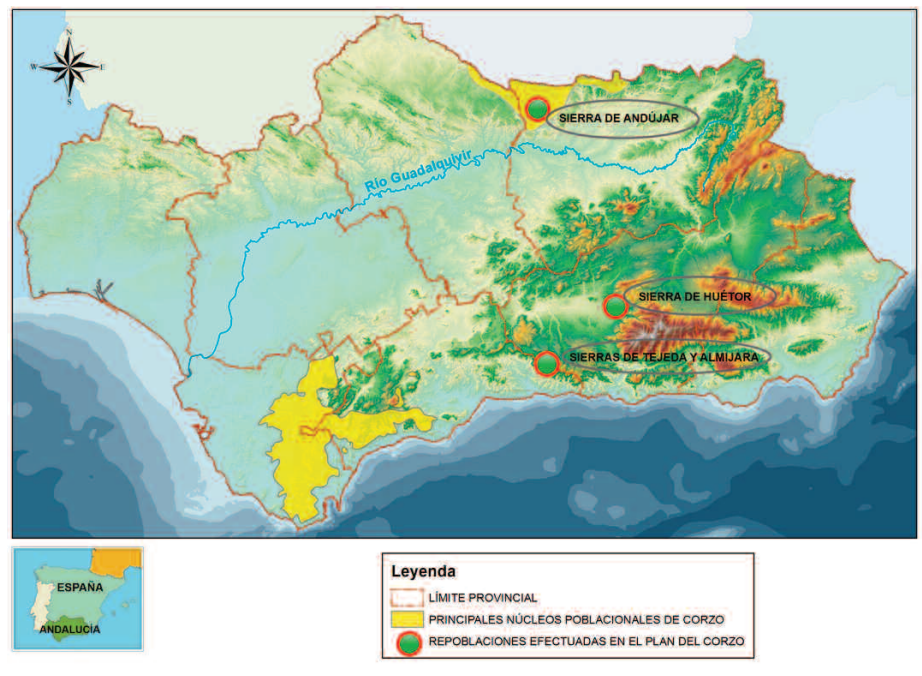

Figura 1. Ubicación de los principales núcleos poblacionales de corzo en Andalucía (modificado de San José 2007) y áreas con repoblaciones efectuadas en el marco del "Plan de Gestión y Reintroducción del Corzo en Andalucía" (Fuente: Consejería de Agricultura, Pesca y Medio Ambiente-Junta de Andalucía).

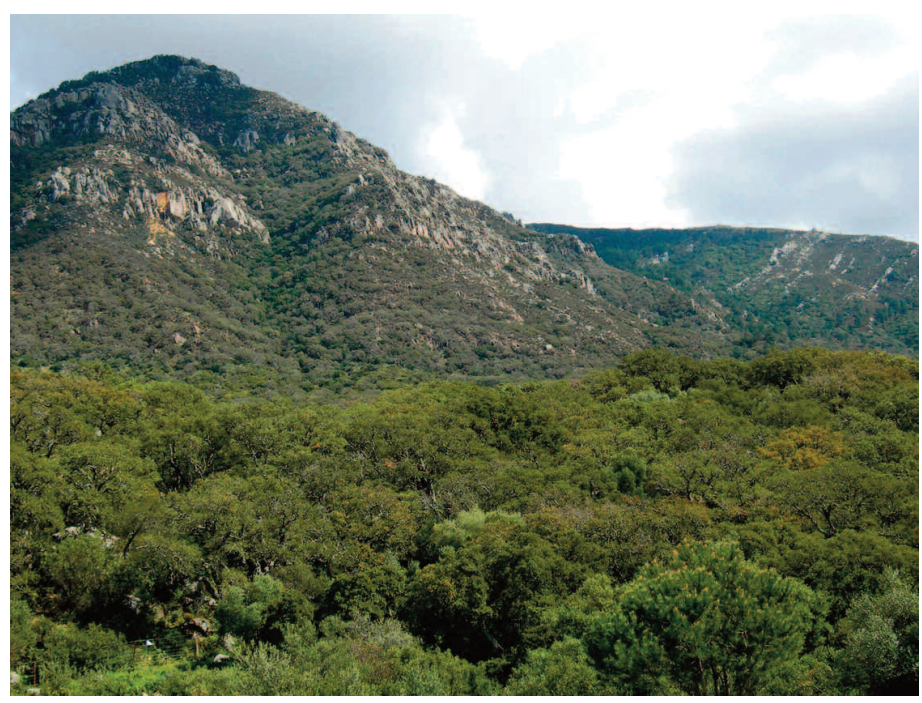

Figura 2. Paisaje del hábitat del corzo en el Área Cinegética Alcornocales donde domina el bosque mediterráneo caracterizado por la presencia de Quercus suber (Autora: Cristina San José).

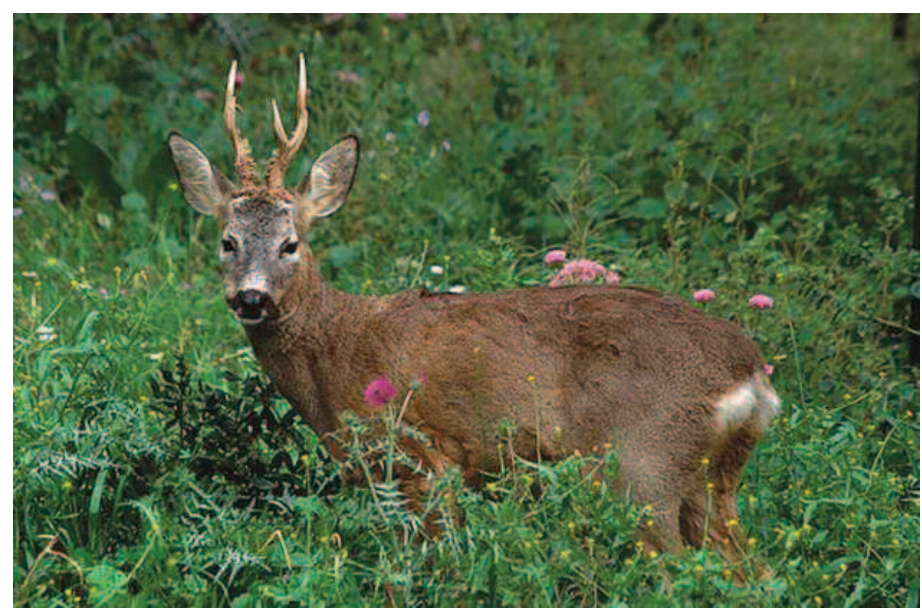

Figura 3. Ejemplar de Corzo Andaluz de la Sierra de Cádiz (Autor: Antonio Atienza). 
anchas que las de los corzos del norte de España y del resto de Europa (Aragón et al. 1998), adaptadas a una vegetación xerofítica mucho más seca y dura. Existen también diferencias significativas en la forma del trofeo del corzo andaluz de Cádiz-Málaga con respecto al resto de corzos de la Península Ibérica y de otras poblaciones europeas, destacando que su cuerna es más corta pero más gruesa, que hay significativamente más ejemplares de corzo andaluz cuyo trofeo presenta más de 6 puntas, y que la apertura de su cuerna es significativamente menor que en los trofeos de corzos del resto de España y Europa (Cassinello et al. 2009).

A nivel genético existen diferencias significativas entre los corzos de la mitad norte y la mitad sur de la Península Ibérica, con una segregación geográfica y genética dentro de la "subpoblación sur", encuadrándose los corzos de las Sierras de Cádiz-Málaga en una población genéticamente independiente, lo que corrobora las diferencias encontradas a nivel morfológico y la existencia de una raza o ecotipo singular propio de éstas (Lorenzini et al. 2003).

Las poblaciones andaluzas de corzo constituyen la última frontera de la especie en su límite suroccidental de distribución mundial, ocupando un hábitat mediterráneo donde el prolongado estiaje actúa como época crítica y mantiene a las poblaciones por debajo del potencial que presenta la especie en el resto de Europa. La densidad media de las poblaciones de corzo en Cádiz-Málaga se sitúa en los últimos 10 años en torno a los 8-9 ejemplares $/ \mathrm{km}^{2}$, con un rango que oscila según la zona desde valores inferiores a 1 corzo/ $\mathrm{km}^{2}$ hasta los 15 ejemplares $/ \mathrm{km}^{2}$, alcanzando incluso densidades superiores a los 20 corzos $/ \mathrm{km}^{2}$ en algunas fincas privadas, con una gestión orientada a la producción de corzo (estimas realizadas mediante conteo de excrementos: Consejería de Medio Ambiente 2007; San José 2009; Duarte et al. 2012; y mediante itinerarios de censos (distance sampling): Junta de Andalucía 2012). En cuanto a la relación de sexos, debería tender al 1:1 dada la territorialidad y estructura social de la especie, pero su estatus de "vulnerable" en Andalucía (Braza et al. 2001) ha propiciado un sesgo en la presión cinegética hacia los machos, por lo que la proporción de sexos se sitúa actualmente en 1.6 hembras por macho (Junta de Andalucía 2012).

En el caso de la Sierra Morena andaluza, las estimas poblacionales de corzo realizadas a finales de los 90 en la provincia de Jaén, mediante conteo de indicios y algunos escasos avistamientos (Azorit y Muñóz-Cobo 1997), constataron entonces una densidad muy por debajo de 1 ejemplar $/ \mathrm{km}^{2}$. La información proveniente de los actuales avistamientos de corzo en las provincias de Córdoba y Jaén hace pensar que el área de distribución de la especie se ha podido incrementar ligeramente en ambas provincias, pero en cualquier caso los niveles de abundancia siguen siendo muy bajos.

\section{Evolución en las capturas de corzo}

Las variaciones en las capturas de corzo son indicativas de la evolución de las densidades poblacionales, y en el caso de Andalucía, dado el estatus expuesto en el apartado anterior, la mayor parte de las capturas de corzo tienen lugar en la provincia de Cádiz (más del $95 \%$ ). A su vez, más del $95 \%$ de los corzos cazados en Cádiz corresponden al Área Cinegética Alcornocales (Junta de Andalucía 2012), que cuenta con una superficie total próxima a las 200000 ha y está incluida en un $94 \%$ en la provincia de Cádiz, correspondiendo el resto a la provincia de Málaga. Las capturas de corzo en el Área Cinegética Alcornocales constituyen por tanto un indicador representativo de la evolución de la población total de Corzo Andaluz originario de las Sierras de Cádiz-Málaga.

Además, como se expone a continuación, el análisis de las capturas de corzo en las modalidades de caza de rececho y batida en el Área Cinegética Alcornocales pone de manifiesto otros aspectos de la evolución de las poblaciones de corzo, vinculados a la gestión local que llevan a cabo algunos cotos privados de caza, con el objetivo primordial de maximizar la producción de corzos.

De acuerdo con el "Decreto 182/2005, de 26 de julio, por el que se aprueba el Reglamento de Ordenación de la Caza" (Junta de
Andalucía 2005) y el "Plan de Caza del Área Cinegética Alcornocales" (Junta de Andalucía 2012), la batida queda definida en los siguientes términos para el corzo en el Área Cinegética Alcornocales: cacería organizada con puestos fijos que se celebra con un número de cazadores igual o superior a nueve; se podrá autorizar la batida con un máximo de 30 puestos y un número máximo de perros de 1 por cada 2 ha; para evitar daños a corzas y corcinos, los perros serán pequeños, de rastro, nunca de presa, e irán en compañía del batidor, en ningún caso formando reala o dispersos; se autorizará una sola batida cada 500 ha de acotado, ampliable excepcionalmente cada 250 ha en casos en los que las condiciones exigidas para esta modalidad por el "Plan de Caza del Área Cinegética Alcornocales" se cumplan sobradamente y las características del coto y de la población de corzos lo permitan.

Actualmente la batida de corzo se practica en Andalucía solo en el Área Cinegética Alcornocales, y en concreto en fincas que cumplen con unos requisitos de "Buenas Prácticas Cinegéticas" y una densidad poblacional de corzo superior a 9 ejemplares $/ \mathrm{km}^{2}$, exigidos por el "Plan de Caza del Área Cinegética Alcornocales" (Junta de Andalucía 2012).

En base a los datos procedentes de los precintos de caza de los corzos cazados y de las Actas de las batidas de corzo, se ha analizado el número de corzos cazados en rececho y batida por temporada en el Área Cinegética Alcornocales (Figs. 4 y 5).

En el caso de los corzos cazados en rececho (Fig. 4), se mantiene una fluctuación a lo largo de los últimos 10 años en torno a 200-250 ejemplares por temporada, con un repunte de 328 corzos cazados en rececho en la última temporada 2011/2012.

Respecto al número de corzos cazados en batida, la evolución es más llamativa (Fig. 5). Las batidas de corzo se reiniciaron en el Parque Natural Los Alcornocales en la primavera del año 2000, tras

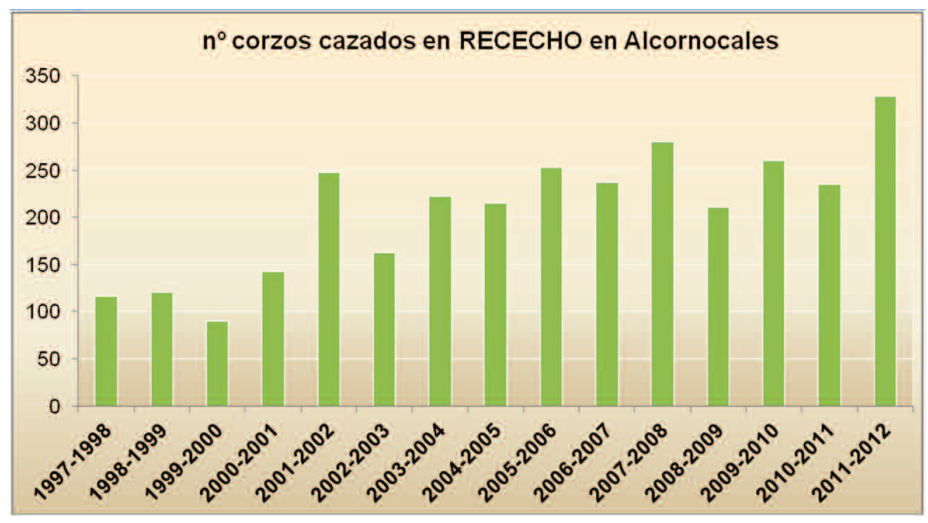

Figura 4. Evolución de las capturas de corzo realizadas en "rececho" en el Área Cinegética Alcornocales (Fuente datos: Consejería de Agricultura, Pesca y Medio Ambiente-Junta de Andalucía).

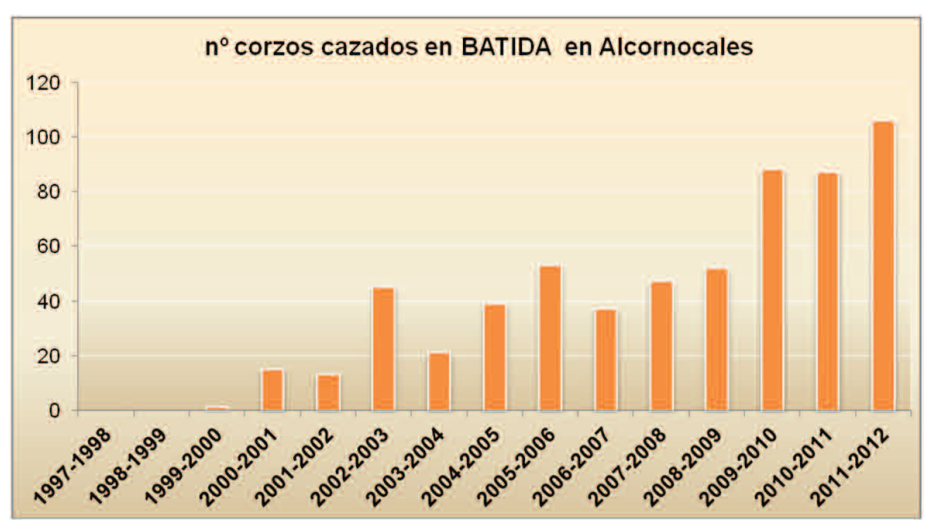

Figura 5. Evolución de las capturas de corzo realizadas en "batida" en el Área Cinegética Alcornocales (Fuente datos: Consejería de Agricultura, Pesca y Medio Ambiente-Junta de Andalucía). 
aproximadamente una década sin practicar esta modalidad de caza en Cádiz (conocida en otras épocas también como "ganchos de corzo"). Este tipo de batidas de caza cesaron en las Sierras de Cádiz-Málaga a principios de los años 90 , por un lado, debido al auge de las monterías sobre otras especies de caza mayor (principalmente ciervo), y por otro, a la disminución paralela que sufrieron los efectivos de corzo en esa época. Ante la disminución drástica de esta población a finales de los 80 , la Administración puso en marcha los primeros estudios sobre el corzo de Cádiz-Málaga, con objeto de evaluar el estado de sus poblaciones y definir las premisas fundamentales para su conservación y manejo (Braza et al. 1994, 2004). En ese momento, y a lo largo de aproximadamente una década, se interrumpieron las batidas de corzo en base a la baja densidad de población estimada a principios de los 90 para las Sierras de Cádiz-Málaga ( 5 corzos $/ \mathrm{km}^{2}$, estimas realizadas mediante transectos de conteo de excrementos; Braza et al. 1994) y al impacto negativo que los perros tienen sobre la especie, particularmente durante la primavera (época en la que tiene lugar la paridera del corzo y se celebran las batidas de corzo en Cádiz).

El número de corzos cazados en batida por temporada en el Área de Alcornocales entre los años 2002 y 2009 fluctuó en torno a un valor medio de 42 ejemplares (Fig. 5), pero en la primavera de 2010 esa cifra se duplicó, y en las tres últimas temporadas (2009-2012) el número anual de corzos cazados en batida ha sobrepasado los 80 corzos, con un repunte hasta los 106 corzos cazados en esta modalidad durante la última temporada 2011-2012.

Si se analizan con detalle los datos de las batidas (Fig. 6), se observa que a lo largo de los últimos 12 años, tanto el número de fincas que dieron batidas de corzo, como el número total de batidas de corzo por temporada, fluctuaron muy poco $\left(n^{\circ}\right.$ medio fincas con batidas $=4.90 \pm 1.22 ; n^{\circ}$ medio batidas por temporada $=8.20 \pm$ 2.09), lo que contrasta con el progresivo incremento observado en el número de corzos cazados en batida a lo largo de esos años. Esto es así, porque el incremento en el número de corzos cazados en batida responde no a un incremento generalizado del corzo y de sus capturas en el Área de Alcornocales, si no a un incremento local del corzo y de sus capturas en esas 3-6 fincas por temporada que han dado batidas de corzo, y que corresponden a cotos privados de caza mayor donde se está llevando a cabo una gestión dirigida a maximizar la producción de corzos. Los puntos clave de esta gestión orientada a potenciar el corzo son: mantener niveles relativamente bajos de abundancia de ciervo (inferiores a 15 ejemplares $/ \mathrm{km}^{2}$ ), realizar mejoras de hábitat para favorecer al corzo (rozas en la vegetación, siembras, comederos y bebederos con acceso limitado para uso exclusivo del corzo), y llevar a cabo un control continuado sobre el cochino asilvestrado (muy difícil de erradicar en la zona) y sobre potenciales depredadores, como el zorro. Ello ha conducido a densidades de corzo que como se indicaba en el apartado anterior, alcanzan en algunas fincas privadas cerradas valores de 15-20 ejemplares/km² (San José 2009; Junta de Andalucía 2012)

\section{Objetivos y estrategia actual para la conservación y gestión del corzo en Andalucía}

En Andalucía la relativamente baja densidad de población de corzo hace que actualmente la gestión de la especie tenga un carácter fundamentalmente de conservación para garantizar el futuro de las poblaciones y potenciar su desarrollo y expansión colonizadora hacia nuevas áreas. Por otro lado, el corzo como especie cinegética ha visto incrementado su valor cinegético, y en el caso de Andalucía aún más, al describirse un ecotipo singular propio de las Sierras de Cádiz-Málaga con un trofeo diferenciado (Cassinello et al. 2009).

Entre las principales amenazas de conservación actualmente para el corzo en Andalucía cabe destacar dos:

- La hibridación del ecotipo de Corzo Andaluz originario de las Sierras de Cádiz-Málaga con corzos no autóctonos, ya que los ejemplares autóctonos de una población son los mejor adaptados a vivir en los ambientes que le son propios, y la hibridación

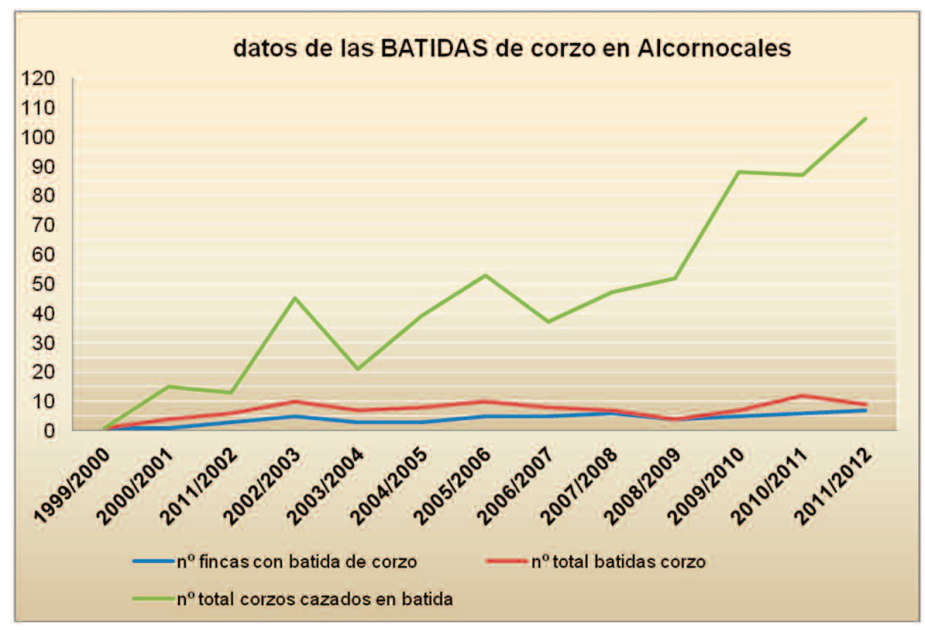

Figura 6. Datos de las "batidas" de corzo en el Área Cinegética Alcornocales (Fuente datos: Consejería de Agricultura, Pesca y Medio Ambiente-Junta de Andalucía).

con ejemplares no autóctonos conduciría a la pérdida de adaptaciones locales que han tardado mucho tiempo en conseguirse.

- El bajo tamaño poblacional de algunas subpoblaciones, y su aislamiento geográfico por barreras tales como mallas cinegéticas o infraestructuras viales, lo que podría conducir a una pérdida de variabilidad genética, y en consecuencia a un declive de las poblaciones.

Partiendo de estas premisas, la gestión del corzo en Andalucía se articula alrededor de dos objetivos principales:

- Conservar el ecotipo de Corzo Andaluz propio de las sierras de Cádiz-Málaga.

- Garantizar el buen estado de conservación del hábitat que ocupa actualmente la especie en Andalucía y mejorar las áreas potencialmente buenas para su colonización natural o mediante repoblaciones planificadas.

En este sentido, desde hace tiempo se llevan aplicando en Andalucía medidas de gestión encaminadas a favorecer al corzo, particularmente en las Sierras de Cádiz-Málaga, tanto en lo referente a la mejora de las condiciones del hábitat como a aspectos de gestión de poblaciones relacionados con la interacción del corzo con otros ungulados silvestres y domésticos (Braza et al. 1994; Consejería de Medio Ambiente 2007):

\section{Gestión del Hábitat}

El agua es uno de los factores más limitantes para la presencia de corzo en Andalucía, requiriendo agua limpia y preferiblemente que no esté encharcada. Por ello, de cara a garantizar la presencia de corzo y conseguir poblaciones estables en la región, en primer lugar se deben mantener y tratar de conservar en buen estado las zonas más húmedas, como es el caso de umbrías, barrancos, arroyos, etc. En este área el período más desfavorable para la especie es el verano, y por tanto, la existencia de estas zonas, donde las condiciones ambientales son algo más suaves y la oferta de alimento mayor, supone un claro beneficio sobre el corzo, no sólo por la propia presencia de agua. La limpieza y el mantenimiento permanente de los puntos de agua son fundamentales; las fuentes naturales pueden ser acondicionadas con piedras que impidan que el agua se ensucie y se colmate de fango, y si es necesario, pueden instalarse puntos de agua de origen artificial, con objeto de aumentar la oferta y distribución de los mismos (Fig. 7). Además las fuentes y bebederos deberían estar cercadas con puertas selectivas de acceso exclusivo para corzo ( $0.80 \mathrm{~m}$ de alto por $0.2 \mathrm{~m}$ de ancho), que limiten la entrada de otros ungulados.

Entre las actuaciones de mejoras directas que se pueden ejecutar en el hábitat se encuentran los desbroces o rozas de vegetación, siendo una medida sencilla de manejo que da óptimos 


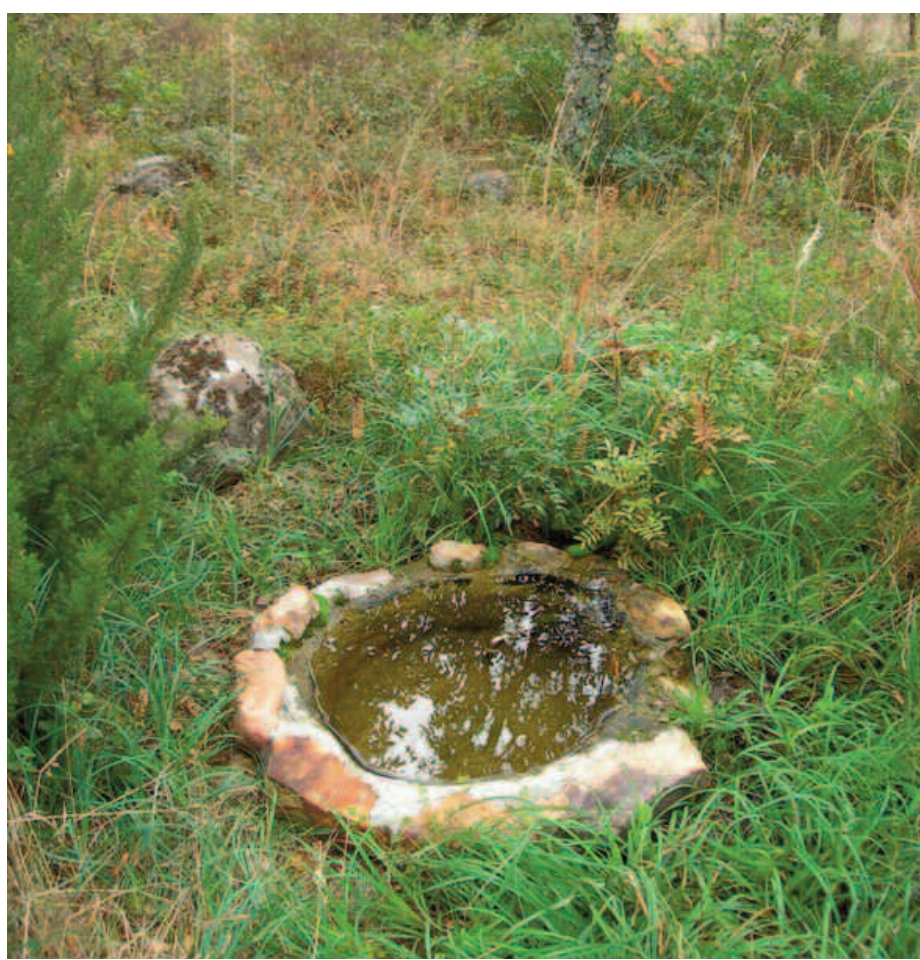

Figura 7. Bebedero acondicionado para corzo (Autora: Cristina San José).

resultados donde la vegetación arbustiva tiene cierta altura y los brotes tiernos quedan fuera del alcance del corzo. Mediante esta medida se aprovecha la capacidad de regeneración de las especies mediterráneas, que tras el desbroce producen brotes de gran palatabilidad y muy ricos en nutrientes. Se recomienda la roza manual de superficies pequeñas (0.5-1 ha) de contorno irregular, que entremezclen zonas abiertas y cubiertas.

En algunos lugares donde la oferta alimenticia sea menor, se pueden asimismo sembrar parcelas con especies apetecibles para el corzo (mezcla de leguminosas con algún cereal). Estas siembras tendrán mayor eficacia para el corzo si se instalan limitando el acceso al resto de ungulados mediante cercados de protección, de forma que solamente pueda entrar el corzo mediante puertas selectivas similares a las de los puntos de agua.

\section{Gestión de Poblaciones}

Un factor relevante a la hora de entender la desaparición del corzo en algunas áreas andaluzas es la proliferación del ciervo, la introducción de otros ungulados silvestres, como gamos y muflones, y la presencia de ganado doméstico (especialmente caprino); todas estas especies compiten con ventaja frente al corzo, tanto de forma directa por tener mayor tamaño, como por una competencia indirecta por el espacio; también la excesiva abundancia de jabalí (o cochino asilvestrado) tiene un efecto negativo sobre el corzo, debido a la transformación que hacen del hábitat y a la predación que pueden ejercer sobre las crías de corzo (Consejería de Medio Ambiente 2007). Por todo ello, se debe evaluar la capacidad de carga del hábitat y adecuar la densidad de ungulados silvestres y domésticos a unos niveles compatibles con la presencia de corzo.

Para prevenir la hibridación de las poblaciones autóctonas de corzo, hay que evitar las repoblaciones con corzos no autóctonos. En este sentido, siguiendo los criterios de la Unión Internacional para la Conservación de la Naturaleza (IUCN 1987), y partiendo de los resultados de los estudios llevados a cabo sobre el Corzo Andaluz en los últimos 20 años (ver apartado "Caracterización, distribución y estatus de las poblaciones andaluzas de corzo"), los corzos para repoblaciones en Andalucía deberían pertenecer a la población lo más cercana posible, y se deberían tener en cuenta los siguientes criterios:

Utilizar ejemplares de Corzo Andaluz originarios de las Sierras de Cádiz-Málaga para repoblaciones al sur del Río Guadalquivir.
Utilizar ejemplares de la subpoblación ibérica centro-meridional (Ciudad Real-Extremadura-Montes de Toledo) para repoblaciones al norte del Río Guadalquivir.

En relación con las repoblaciones con corzo andaluz originario de las Sierras de Cádiz-Málaga, la administración autonómica prevé la puesta en marcha de un protocolo de certificación genética de calidad de origen del corzo andaluz, usando las técnicas de análisis genético disponibles capaces de discriminar este ecotipo de corzo del resto de corzos de España y Europa (Lorenzini et al. 2003; Alasaad y Soriguer 2012, datos sin publicar).

En cuanto a la gestión cinegética, con carácter general, se aconseja una gestión orientada hacia la obtención de trofeos, primando la calidad frente a la cantidad, y para conservar el estado natural y silvestre de las poblaciones de corzo, se debe evitar interferir en todo lo posible en el que suele ser el periodo más crítico de su ciclo biológico: la reproducción. Teniendo en cuenta que la tasa reproductiva del corzo en Andalucía es inferior a 1 cría/hembra (datos de avistamientos incluyendo hembras jóvenes de 1 año: Braza et al. 1994; Consejería de Medio Ambiente 2007; San José 2009), valor similar al de otras poblaciones centro-meridionales de la Península Ibérica (1 cría por hembra reproductiva en bosques de quercíneas de Las Villuercas, Cáceres; Mateos-Quesada y Carranza 2000), pero por debajo de la media descrita para la mayor parte de poblaciones de corzo del centro y norte de Europa (rango: 1.55-2.23 crías por hembra reproductiva; Andersen et al. 1998), se deberían minimizar las causas de estrés durante el periodo de cría en las poblaciones más meridionales de corzo, primando la caza al rececho y evitando o minimizando la caza con perros en primavera.

Partiendo de estos aspectos básicos, y de que la planificación cinegética en Andalucía se basa en una división territorial en 23 áreas cinegéticas establecidas por hábitats homogéneos (Junta de Andalucía 2007), el "Plan de Caza del Área Cinegética Alcornocales" (Junta de Andalucía 2012), primer plan comarcal de caza aprobado en Andalucía, establece las directrices para la explotación cinegética del corzo en los siguientes términos: "la posibilidad de caza deberá ser siempre moderada para no comprometer la viabilidad de la población; con carácter general, la posibilidad no debe ser mayor del 20 \% de la población adulta total cuando la densidad sea de más de 9 ejemplares $/ \mathrm{km}^{2}$. Cuando la densidad oscile entre 6-9 ejempla$\mathrm{res} / \mathrm{km}^{2}$ se considera adecuada una posibilidad del $15 \%$, y del 10 $\%$ cuando la densidad sea inferior a 6 ejemplares $/ \mathrm{km}^{2}$. Dicha extracción se deberá realizar ajustándola a la relación de sexos y la estructura poblacional adecuadas, con tendencia a cazar el mismo número de machos que de hembras. El plan de caza de corzos y corzas se deberá establecer en el PTC en función de la densidad y relación de sexos, y se procurará que los ejemplares reúnan las siguientes características: ejemplares adultos con trofeo plenamente desarrollado, machos selectivos (viejos, enfermos, heridos o con trofeo malformado) y hembras adultas, enfermas o heridas. No se aconseja disparar en ningún caso sobre ejemplares jóvenes con buen futuro".

Respecto al estado sanitario de los corzos en Andalucía, puede considerarse que el riesgo procede de factores externos ya que las bajas densidades de población, y el comportamiento solitario de la especie, no propician el desarrollo de brotes epidemiológicos, si bien la especie es más sensible que otros cérvidos a los procesos parasitarios (León et al. 1994; Gortázar y Fernández de Luco 2005). Por ello, es muy importante el control sanitario del ganado doméstico y el control cinegético de las poblaciones de otros ungulados silvestres que pueden transmitir enfermedades a los corzos. Además, particularmente en los años de sequía, es fundamental el acondicionamiento de los puntos de agua exclusivos para el corzo antes de empezar el verano. El seguimiento sanitario de las poblaciones de corzo está incluido en el Programa de Vigilancia de la Fauna Silvestre en Andalucía (Consejería de Agricultura, Pesca y Medio Ambiente, Junta de Andalucía).

En lo referente a depredadores carnívoros, el corzo es sensible, particularmente en edades tempranas, al impacto de los zorros y perros asilvestrados, por lo que el control de éstos se debería intensificar previamente a la época de cría del corzo. 
Además, para favorecer la expansión del corzo e incrementar el área de distribución de la especie en Andalucía, la Junta de Andalucía puso en marcha en 2009 el "Plan de Gestión y Reintroducción del Corzo en Andalucía", orientado al refuerzo de las poblaciones andaluzas de corzo que presentan niveles bajos de abundancia, a la reintroducción de la especie en áreas andaluzas idóneas donde ésta se extinguió en tiempos recientes, y a la ejecución de actuaciones encaminadas a mejorar la capacidad de acogida del hábitat, primando al corzo frente a otras especies cinegéticas de caza mayor.

Como se indicaba más arriba, en 2011 y 2012 se llevaron a cabo repoblaciones de corzo en la Sierra de Andújar (Jaén), Sierra de Huétor (Granada), y Sierras de Tejeda-Almijara (Málaga) (Fig. 1, Fig. 8 y Tabla 1), en áreas idóneas donde la especie se extinguió en tiempos recientes. En concreto se introdujeron 21 ejemplares (5 machos y 16 hembras) en cercados temporales de aclimatación, con una superficie entre 6 y 15 ha. Los ejemplares repoblados en Sierra Morena proceden de los Montes de Toledo (núcleo genético centro-meridional de la Península Ibérica; Lorenzini et al. 2003), y los reintroducidos en la Sierra de Huétor y en las Sierras de TejedaAlmijara son originarios de la Sierra de Cádiz (núcleo genético de las Sierras de Cádiz-Málaga; Lorenzini et al. 2003)

El éxito de aclimatación de los ejemplares repoblados ha sido del $71 \%$, debiéndose las bajas a la alta sensibilidad de los corzos al estrés post-captura. Para minimizar los efectos de este estrés se aplican tratamientos veterinarios específicos para el manejo, traslado y aclimatación de los ejemplares. Dichos tratamientos, particularmente los anestésicos (Flores et al. 2007), se han testado y puesto a punto sobre ejemplares de la Estación de Referencia del Corzo Andaluz (Alcalá de Los Gazules-Cádiz, Consejería de Agricultura, Pesca y Medio Ambiente-Junta de Andalucía).

En los cercados de aclimatación se llevó a cabo un seguimiento de los corzos repoblados mediante la observación directa, y a través de cámaras de foto-trampeo instaladas en la proximidad de comederos y bebederos. Estas cámaras (de fácil colocación y coste no excesivo) permiten comprobar si los ejemplares trasladados se aclimatan al nuevo territorio y registrar aspectos interesantes de comportamiento, tales como las posibles agresiones por parte de los machos adultos hacia los machos juveniles cuando se inicia el periodo territorial. Dado el carácter huidizo de los corzos y la vegetación natural presente en los cercados de aclimatación, las cámaras se han revelado como la herramienta más eficaz para realizar el seguimiento de los ejemplares translocados / reintroducidos, ya que el corzo al ser territorial acude a lugares fijos para beber y alimentarse, y es en estos lugares donde pueden ser registrados por las cámaras de foto-trampeo. Estas cámaras han permitido detectar en sus primeros días de vida a las crías nacidas en los cercados de aclimatación (Fig. 9): 2 en el cercado de la Sierra de Andújar, 7 en el cercado de las Sierras de Tejeda-Almijara, y 2 en el cercado de la Sierra de Huétor. La diferencia entre cercados en cuanto al número de crías nacidas estriba en la mayor o menor presencia de hembras jóvenes (de menos de un año de edad) entre los ejemplares inicialmente reintroducidos.

En junio de 2012 se abrieron puertas selectivas en el cercado de la Sierra de Tejeda-Almijara, con objeto de favorecer el inicio de la dispersión, habiéndose marcado un macho juvenil con un collar radio-emisor GPS-GSM, que ha permitido comprobar su entrada y salida regular del cercado a lo largo de los últimos 6 meses. Las puertas selectivas abiertas en el cercado tienen una dimensión adaptada al tamaño de los corzos $(0.80 \mathrm{~m}$ de alto por $0.2 \mathrm{~m}$ de ancho), limitando el acceso de ungulados de mayor tamaño como el ciervo, el jabalí o la cabra montés, presentes en la zona. En los otros dos cercados de aclimatación de la Sierra de Andújar y Sierra de Huétor se ha facilitado la conexión desde los cercados iniciales de aclimatación hacia otros subcercados adyacentes, originalmente disponibles, con objeto de incrementar el espacio para evitar la competencia entre machos y consolidar un mayor tamaño de población antes de llevar a cabo la apertura hacia el exterior y el inicio de la dispersión de los corzos.

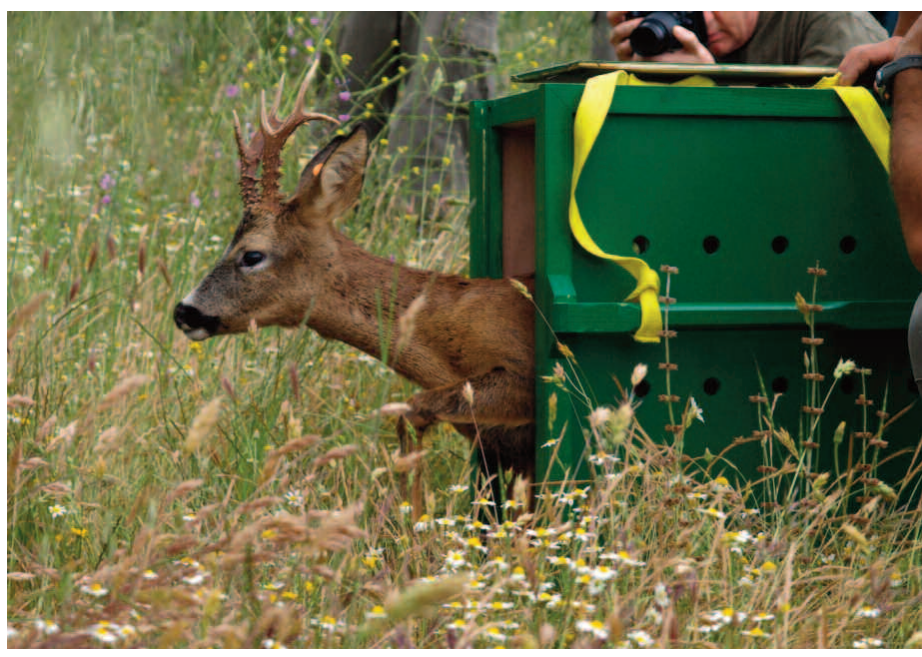

Figura 8. Suelta de macho de corzo repoblado en el cercado de aclimatación ubicado en la Sierra de Huétor (Granada) (Autor: Francisco Javier Galindo).

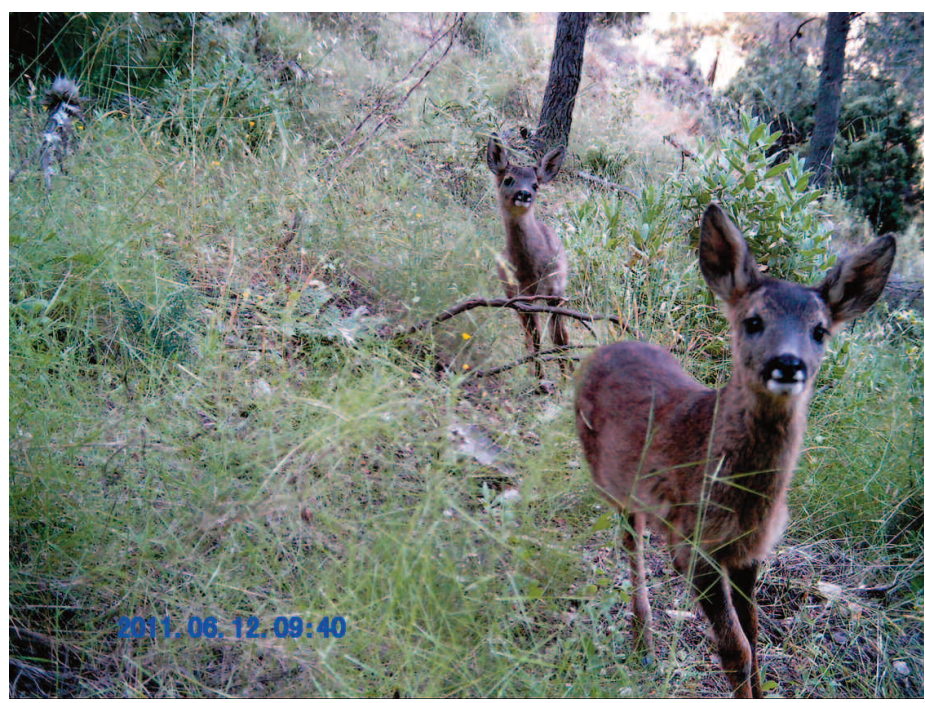

Figura 9. Imagen de crías de corzo obtenida por "foto-trampeo" en el cercado de aclimatación ubicado de las Sierras de Tejeda-Almijara (Málaga).

Tabla 1. Repoblaciones de corzo efectuadas en 2011-2012 en el marco del "Plan de Gestión y Reintroducción del Corzo en Andalucía" (Fuente: Consejería de Agricultura, Pesca y Medio Ambiente-Junta de Andalucía).

\begin{tabular}{cccc}
\hline FECHA & N $^{\circ}$ CORZOS & PROCEDENCIA & DESTINO \\
\hline $20 / 01 / 2011$ & 4 & Montes de Toledo & Sierra de Andújar, \\
& $\begin{array}{c}(1 \text { macho/ } \\
3 \text { hembras })\end{array}$ & & JAÉN
\end{tabular}

\begin{tabular}{|c|c|c|c|}
\hline 08/03/2011 & $\begin{array}{c}5 \\
\text { (1 macho/ } \\
4 \text { hembras) }\end{array}$ & Sierra de Cádiz & $\begin{array}{l}\text { Sierras de Tejeda- } \\
\text { Almijara, MÁLAGA }\end{array}$ \\
\hline $30 / 03 / 2011$ & $\begin{array}{c}5 \\
\text { (1 macho/ } \\
4 \text { hembras) }\end{array}$ & $\begin{array}{c}\text { Sierra de Cádiz } \\
\text { (Estación de Referencia } \\
\text { del Corzo andaluz) }\end{array}$ & $\begin{array}{l}\text { Sierra de Huétor, } \\
\text { GRANADA }\end{array}$ \\
\hline 01/06/2011 & $\begin{array}{c}1 \\
(1 \text { macho })\end{array}$ & Sierra de Cádiz & $\begin{array}{l}\text { Sierra de Huétor, } \\
\text { GRANADA }\end{array}$ \\
\hline $11 / 10 / 2011$ & $\begin{array}{c}4 \\
(1 \text { macho/ } \\
3 \text { hembras) }\end{array}$ & Montes de Toledo & $\begin{array}{c}\text { Sierra de Andújar, } \\
\text { JAÉN }\end{array}$ \\
\hline 01/03/2012 & $\begin{array}{c}2 \\
\text { (2 hembras) }\end{array}$ & $\begin{array}{c}\text { Sierra de Cádiz } \\
\text { (Estación de Referencia } \\
\text { del Corzo andaluz) }\end{array}$ & $\begin{array}{l}\text { Sierra de Huétor, } \\
\text { GRANADA }\end{array}$ \\
\hline
\end{tabular}




\section{Agradecimientos}

Todos los estudios sobre el corzo andaluz de las Sierras de Cádiz-Málaga mencionados en este trabajo han sido financiados por el Gobierno de la Junta de Andalucía. En la obtención de datos han colaborado la mayor parte de los titulares y gestores de los cotos privados de caza mayor de las provincias de Cádiz y Málaga, así como los guardas de montes públicos y privados, personal de la Agencia de Medio Ambiente y Agua de Andalucía, del Parque Natural Los Alcornocales, e investigadores de la Estación Biológica de Doñana (CSIC) y del Instituto de Investigación en Recursos Cinegéticos (IREC, CSIC-UCLM-JCCM). Para actualizar la información sobre las capturas de corzo se ha contado con el apoyo del personal de de la Consejería de Agricultura, Pesca y Medio Ambiente de la Junta de Andalucía tanto en Servicios Centrales como en las Delegaciones Territoriales. La cartografía de este artículo ha sido elaborada por Noelia García León y Maria Luisa Sillero (Agencia de Medio Ambiente y Agua de Andalucía).

\section{Referencias}

Andersen, R., Gaillard, J.M., Liberg, O., San José, C., 1998. Variation in lifehistory parameters. En: Andersen, R., Duncan, P. and Linnell, J.D.C. (eds.), The European Roe Deer: The Biology of Success, pp 285-307. Scandinavian University Press, Oslo. Norway.

Aragón S. 1993. El Corzo (Capreolus capreolus) en Cádiz. Tesis Doctoral. Universidad de Sevilla, España.

Aragón, S., Braza, F., San José, C. 1995a. Características morfológicas de los corzos (Capreolus capreolus) de las sierras de Cádiz-Málaga. Doñana Acta Vertebrata 22:51-64.

Aragón, S., Braza, F., San José, C. 1995b. Socioeconomic, physiognomic, and climatic factors determining the distribution pattern of roe deer (Capreolus capreolus) in Spain. Acta Theriologica 40(1):37-43.

Aragón, S., Braza, F., San José, C. 1998. Variation in skull morphology of roe deer (Capreolus capreolus) in Werstern Europe. Journal of Mammalogy 79:131-140.

Azorit Casas C. Muñoz-Cobo Rosales J. 1997. Distribución y abundancia del corzo (Capreolus capreolus L. 1758) en la provincia de Jaén. Doñana Acta Vertebrata 24(1-2):181-190.

Braza, F., San José, C., Aragón, S., Delibes, J.R. 1994. El corzo andaluz. Consejería de Agricultura y Pesca-Junta de Andalucía, Sevilla. España.

Braza, F., Soriguer, R., Aragón, S., San José, C. 2001. Corzo Capreolus capreolus (Linnaeus, 1758). En: Consejería de Medio Ambiente-Junta de Andalucía (ed.), Libro rojo de los vertebrados amenazados de Andalucía, pp. 266-267, Sevilla. España.

Braza, F., San José, C., López, M. 2004. El corzo en Los Alcornocales. Mapa de Calidad de Hábitats. Consejería de Obras Públicas-Junta de Andalucía, Sevilla, España.

Carranza, J. 2010. Ungulates and their management in Spain. En: Apollonio, M., Andersen, R. and Putman, R. (eds.), European Ungulates and their management in the 21st century, pp. 421-422. Cambridge University Press, Cambridge, UK.

Cassinello, J., Caro, J., Delibes-Mateos,M., San José, C., Tena, M.A. 2009. A morphobiometric study of the Andalusian roe deer antlers: Comparison with other roe deer populations in Spain and Europe. Presentations at the 9th European Roe Deer Congress, Edinburgh, Scotland.

Consejería de Medio Ambiente 2007. Manual de conservación y gestión del corzo andaluz. Manuales de conservación de la naturaleza N4. Consejería de Medio Ambiente-Junta de Andalucía, Sevilla, España.

Duarte, J., Farfán, M.A., Vargas, M. 2012. Situación de las poblaciones de corzo andaluz (Capreolus capreolus) en las sierras occidentales de Málaga. Galemys 24:35-45.

Flores, L., Rodríguez, P., San José, C., Pérez C., Dorado, A., Oliveros, F. 2007. Capture and chemical inmobilization of roe deer (Capreolus capreolus) in SW Spain. Presentations at the 8th European Roe Deer Congress, Velenje, Slovenija, pp. 64.

Gortázar, G., Fernández de Luco, D. 2005. Principales enfermedades del corzo. Boletín de la Asociación del Corzo Español 8:31-36.

IUCN 1987. The IUCN position statement on translocation of living organisms: introductions, re-introductions and restocking. IUCN, Gland, Switzerland.

Junta de Andalucía 2005. Decreto 182/2005, de 26 de julio por el que se aprueba el Reglamento de Ordenación de la Caza. BOJA núm. 154/2005, de 9 de agosto, pp. 36-58.

Junta de Andalucía 2007. Decreto 232/2007, de 31 de julio por el que se aprueba el Plan Andaluz de Caza y se modifica el Reglamento de Ordenación de la Caza aprobado por Decreto 182/2005, de 26 de Julio. BOJA núm. 158/2007, de 10 de agosto, pp. 32-55.

Junta de Andalucía 2012. Plan de Caza del Área Cinegética de Alcornocales. BOJA núm. 156, de 9 de agosto, pp. 56-57. Disponible en: http://www.cma.junta-andalucia.es/medioambiente/site/pcp/Novedades.

León, L., Astorga, R., Cubero, M.J. 1994. Aproximación al estado sanitario de los corzos andaluces. En: Braza F., San José C., Aragón S., Delibes J.R./Consejería de Agricultura y Pesca-Junta de Andalucía (ed.), El corzo andaluz, pp. 85-97, Sevilla. España.

Lorenzini, R., San José, C., Braza, F., Aragón S. 2003. Genetic differenciation and phylogeography of roe deer in Spain, as suggested by mitochondrial DNA and microsatellite analysis. Italian Journal of Zoology 70 (89-99).

Mateos-Quesada, P., Carranza, J. 2000. Reproductive patterns of roe deer in Central Spain. Etologia 8:17-20.

Menor A., Prenda J. 2005. Distribución histórica del corzo (Capreolus capreolus Linnaeus, 1758) en Andalucía (España) en el siglo XIX. Presentations at the 7th European Roe Deer Congress, Jerez, España, pp. 107.

San José, C. 2007. Capreolus capreolus (Linnaeus, 1758). Corzo. En: Palomo, L. J. y Gisbert J., (eds.), Atlas y libro rojo de los mamíferos terrestres de España. pp. 359-361. Dirección General para la Biodiversidad-SECEM-SECEMU, Madrid, España.

San José, C. 2009. Gestión del Corzo en Andalucía. En: Sáenz de Buruaga, M. y Carranza, J. (eds.), Gestión Cinegética en los ecosistemas mediterráneos (Vol. II) pp. 287-299. Consejería de Medio Ambiente-Junta de Andalucía, Sevilla, España. 